\title{
A Probabilistic Transmission Dynamic Model to Assess Indoor Airborne Infection Risks
}

\author{
Chung-Min Liao, ${ }^{1 *}$ Chao-Fang Chang, ${ }^{1}$ and Huang-Min Liang ${ }^{1}$
}

\begin{abstract}
The purpose of this article is to quantify the public health risk associated with inhalation of indoor airborne infection based on a probabilistic transmission dynamic modeling approach. We used the Wells-Riley mathematical model to estimate (1) the $\mathrm{CO}_{2}$ exposure concentrations in indoor environments where cases of inhalation airborne infection occurred based on reported epidemiological data and epidemic curves for influenza and severe acute respiratory syndrome (SARS), (2) the basic reproductive number, $R_{0}$ (i.e., expected number of secondary cases on the introduction of a single infected individual in a completely susceptible population) and its variability in a shared indoor airspace, and (3) the risk for infection in various scenarios of exposure in a susceptible population for a range of $R_{0}$. We also employ a standard susceptible-infectious-recovered (SIR) structure to relate Wells-Riley model derived $R_{0}$ to a transmission parameter to implicate the relationships between indoor carbon dioxide concentration and contact rate. We estimate that a single case of SARS will infect 2.6 secondary cases on average in a population from nosocomial transmission, whereas less than 1 secondary infection was generated per case among school children. We also obtained an estimate of the basic reproductive number for influenza in a commercial airliner: the median value is 10.4 . We suggest that improving the building air cleaning rate to lower the critical rebreathed fraction of indoor air can decrease transmission rate. Here, we show that virulence of the organism factors, infectious quantum generation rates (quanta/s by an infected person), and host factors determine the risk for inhalation of indoor airborne infection.
\end{abstract}

KEY WORDS: Airborne infectious disease; basic reproductive number; epidemiology; probabilistic; risk

\section{INTRODUCTION}

Airborne transmission is known to be a route of infection for diseases. About 15 million ( $>25 \%$ ) of 57 million annual deaths worldwide are estimated to be related directly to infectious diseases. Of those infectious-disease-related deaths, 3.96 million annual

\footnotetext{
1 Ecotoxicological Modeling Center, Department of Bioenvironmental Systems Engineering, National Taiwan University, Taipei, Taiwan 10617, ROC.

* Address correspondence to Chung-Min Liao, Ecotoxicological Modeling Center, Department of Bioenvironmental Systems Engineering, National Taiwan University, Taipei, Taiwan 10617, ROC; tel: +886-2-2363-4512; fax: +886-2-2362-6433; cmliao@ntu.edu.tw.
}

deaths $(7 \%)$ occur as a consequence of viral respiratory infections (http://www.who.int/whr/en). Airborne rhinovirus transmitted in office environments, influenza spread on an airplane, tuberculosis transmitted in hospital buildings - are all current examples of airborne infection. ${ }^{(1-5)}$ Control of newly emerging infections like severe acute respiratory syndrome (SARS), bioterrorist-spread infections like smallpox, or pandemic influenza ${ }^{(6)}$ have generated an even more acute sense of the need to analyze airborne infection transmission.

Understanding what determines patterns of infection spread in populations is important for controlling infection transmission. Infectivity data can be analyzed with a mathematical model of airborne 
infections to assess the relationship among infection rate, building ventilation, pulmonary ventilation rate, and exposure duration of the source cases. The risk of infection can be estimated using the exponential dose-response model. ${ }^{(1,2)}$ This model can be expressed as a Poisson probability distribution: $\pi(d)=$ $1-\exp (-r d)$, where $\pi(d)$ is the probability of infection for a given dose $d$ and $r$ is the unique parameter characterizing the process.

Wells ${ }^{(7)}$ used the term "quantum" to indicate the dose of infectious droplet nuclei necessary to initiate infection, based on the assumption that infection requires at least one organism. He used a Poisson distribution to model the average concentrations, recognizing that in low concentrations some occupants in an airspace may escape infection by chance. The Wells-Riley model has been used to estimate the risk of infection with measles and tuberculosis compared with epidemiological data. ${ }^{(8)}$

Myatt et al. ${ }^{(5)}$ have reviewed several related literature and concluded that increased ventilation of buildings could reduce the burden of viral respiratory infections among the occupants and that airborne transmission is an important mode of transmission. Rudnick and Milton ${ }^{(9)}$ suggested that risk of indoor airborne infection transmission could be estimated from $\mathrm{CO}_{2}$ level as a marker for exhaled-breath exposure. With indoor airborne infections, rational disease control requires analysis of the role of building ventilation and other transmission factors by the contact investigation of the cases of airborne infections occurring in an indoor environment. In addition, we also require an understanding of the disease dynamics and risk profiles for different groups in the exposed population. The cost and benefits of alternative control strategies can then be calculated after these are quantified through proper epidemic models. Environmental settings impose both spatial and interindividual heterogeneity that affects the dynamics of disease invasion and spread. ${ }^{(10)}$ In such situations, disease is best studied in a probabilistic rather than a deterministic framework, ${ }^{(11,12)}$ allowing incorporation of chance effects into the model as well as complexity due to the heterogeneity.

A fundamental concept in epidemic dynamics is the basic reproductive number, $R_{0}$, the average number of secondary cases generated by one primary case at the start of the epidemic in a completely susceptible population. ${ }^{(13,14)}$ When $R_{0}>1$ it implies that the epidemic is spreading within a population and that incidence is increasing, whereas $R_{0}<1$ means that the disease is dying out. An average $R_{0}$ of 1 means the disease is in endemic equilibrium within the population.

$R_{0}$ essentially determines the rate of spread of an epidemic and how intensive a policy will need to be to control the epidemic. ${ }^{(15)}$ Measles, for example, is a highly infectious respiratory-transmitted disease and has been estimated to have an $R_{0}$ of approximately $14{ }^{(16)}$ whereas $R_{0}$ for SARS was estimated to be about 3 in nosocomial transmission. ${ }^{(17,18)}$

The objective of this article is to quantify the public health risk associated with indoor airborne infection based on a probabilistic transmission dynamic modeling approach. We used the Wells-Riley mathematical model to estimate (1) the exposure concentrations in indoor environments where cases of inhalation airborne infection occurred based on reported epidemiological data and epidemic curves for influenza and SARS, (2) the basic reproductive number, $R_{0}$, and its variability in a shared indoor airspace, and (3) the risk for infection in various scenarios of exposure in a susceptible population for a range of $R_{0}$. Furthermore, we link the Wells-Riley model derived $R_{0}$ with standard susceptible-infectious-recovered (SIR) model derived transmission parameter to study the relationships between contact rate and exhaled-breath exposure in a ventilated airspace.

\section{MATERIALS AND METHODS}

\subsection{Quantitative Epidemiology of Influenza and SARS Data}

With the detailed epidemiological data from reported cases regarding influenza and SARS associated with epidemic curves (number of cases by date of symptom onset), we estimated the infected probability for influenza and SARS in the absence of interventions and control efforts. SARS, a novel coronavirus, is a recently described illness of humans with a high morbidity and mortality that has been reported locally in Taiwan since April 2003. ${ }^{(18)}$ We have estimated the force of infection based on that estimated infected probability. We reanalyzed data, including infectivity data, human respiratory ventilation under different activities, and environmental conditions. Infectivity data on a weekly basis from the Center for Disease Control, Taiwan, on incidence of influenza based on the elementary school settings from 2003 to 2004 and epidemiology of SARS reported from Taipei Municipal Ho-Ping Hospital on April 24-May 8, 2003 were used in the present study. 
Because of limited knowledge, both from observations and theoretical understanding of transmission in a ventilated airspace, we need to characterize uncertainty and variability. We used a Monte Carlo simulation to quantify our uncertainty concerning reported infection probability and force of infection. We used the Kolmogorov-Smirnov (K-S) statistics to optimize the goodness of fit of distributions. We employed Crystal Ball software (Version 2000.2, Decisioneering, Inc., Denver, CO, USA) to analyze data and to estimate distribution parameters. For this study, 10,000 iterations are sufficient to ensure stability of results.

\subsection{Transmission Model}

Most investigators fit a specific model that summarizes assumptions about the epidemiology of the disease when only times of symptom onset are available. $^{(18,19)}$ We modified the Wells-Riley equation proposed by Rudnick and Milton ${ }^{(9)}$ to model the risk of indoor airborne transmission of infected diseases. The modified Wells-Riley equation expresses infection risk as a function of the fraction of inhaled air that has been exhaled previously by someone in the building (i.e., rebreathed fraction) using $\mathrm{CO}_{2}$ concentration as a marker for exhaled-breath exposure.

Rudnick and Milton ${ }^{(9)}$ proposed a Wells-Riley equation as

$$
\begin{aligned}
P= & \frac{D}{S}=1-\exp \left\{-\frac{i q p t}{Q}\right. \\
& \left.\times\left[1-\frac{V}{Q t}\left(1-\exp \left(-\frac{Q t}{V}\right)\right)\right]\right\},
\end{aligned}
$$

where $P$ is the probability of infection for susceptible population, $D$ is the number of disease cases, $S$ is the number of susceptible, $i$ is the number of infectors, $q$ is the quantum generation rate by an infected person (quanta $\left.\mathrm{S}^{-1}\right), p$ is the breathing rate per person $\left(\mathrm{m}^{3} \mathrm{~s}^{-1}\right), t$ is the total exposure time (seconds), $Q$ is the outdoor air supply rate $\left(\mathrm{m}^{3} \mathrm{~s}^{-1}\right)$, and $V$ is the volume of the ventilated space $\left(\mathrm{m}^{3}\right)$. Equation (1) assumes steady-state exposure; equal host susceptibility; uniform sizes of droplets; uniform ventilation; homogeneous mixing of air; and that elimination of infective particles caused by loss of viability, filtration, settling, and other mechanisms is small compared with removal by ventilation. Then the accumulation rate of quanta is equal to the quantum generation rate minus the rate of quantum removal by ventilation. Rudnick and Milton ${ }^{(9)}$ indicated that Equation (1) is especially useful in poorly ventilated environments when outdoor air supply rates can be assumed constant.

The total $\mathrm{CO}_{2}$ level in the indoor air is contributed from human origin and outdoor air supply. Person-toperson transmission of infectious diseases is through the recirculated air in the ventilation airspace. Therefore, the outdoor air supply rate $(Q)$ can be expressed as functions of the fraction of indoor air that is exhaled-breath $(f)$, people in the ventilated airspace $(n)$, and the breathing rate per person $(p)$, and can be expressed as

$$
Q=\frac{n p}{f} .
$$

Substituting Equation (2) in Equation (1) gives

$$
\begin{aligned}
P= & \frac{D}{S}=1-\exp \left\{-\frac{i q f t}{n}\right. \\
& \left.\times\left[1-\frac{V f}{n p t}\left(1-\exp \left(-\frac{n p t}{V f}\right)\right)\right]\right\} .
\end{aligned}
$$

We use the modified Wells-Riley model shown in Equation (3) to estimate the infection probability.

\subsection{Basic Reproductive Number}

When $i=1$ and $S=n-1, R_{0}$ for an infectious disease in a building environment can be derived from Equation (3) and expressed as,

$$
\begin{aligned}
R_{0}= & (n-1)\left\{1-\exp \left\{-\frac{q f t}{n}\right.\right. \\
& \left.\left.\times\left[1-\frac{V f}{n p t}\left(1-\exp \left(-\frac{n p t}{V f}\right)\right)\right]\right\}\right\} .
\end{aligned}
$$

The basic reproductive number is generally presented as a single point estimate, with no indication of the variability inherent in the estimation of biological parameter. We used the reported epidemic curves of influenza and SARS associated with a probabilistic transmission model, by which we can quantify our uncertainty concerning $R_{0}$ attributable to the large variance of the distribution of the number of secondary cases infected by each source case and the uncertainty in that distribution and the uncertainty due to sampling variability.

\subsection{Total Proportion of Infected Population}

The probability of infection for each susceptible person each day is based on the transmission probabilities for each potentially infected contact. According to Anderson and May, ${ }^{(13)}$ the total proportion of 
infected population during the epidemic $(I)$ is seen to depend only on $R_{0}$ as

$$
I=1-\exp \left(-R_{0} I\right)
$$

Equation (5) is based on the theoretical relationship between epidemic $(I)$ and $R_{0}$ assuming a homogeneous, unstructured population.

\subsection{Risk Analysis}

Risk of infection in a ventilated airspace can be calculated as the proportion of the group expected to be infected multiplied by the conditional probability of epidemic, given basic reproductive number $R_{0}$. This result in a joint probability function describes the probability of an outbreak of diseases in a susceptible population for a range of values of $R_{0}$. Graphic display of the joint probability function also provides a means of assessing how alterations in basic reproductive number $R_{0}$ due to management efforts or natural attenuation would affect the assessment. This can be expressed symbolically as

$$
\Phi\left(R_{0}\right)=P\left(R_{0}\right) P\left(I \mid R_{0}\right),
$$

where $\Phi\left(R_{0}\right)$ is the risk of an outbreak of an epidemic in a susceptible population for a range of $R_{0}$, $P\left(R_{0}\right)$ is the probability density function of $R_{0}$, and $P\left(I \mid R_{0}\right)$ is the conditional probability of the infection, given basic reproductive number $R_{0}$. To show how Equation (6) can be employed in airborne infection risk assessment, we consider several environmental settings: influenza in an aircraft environment and SARS transmission in the hospital and elementary school. We analyzed aircraft and classroom in the elementary school as a single room and the hospital as an entire floor of a large building, representing an independently ventilated shared airspace. As noted by Rudnick and Milton, ${ }^{(9)}$ we assume that one individual develops illness at the start of a school or workday and exposes others for a definite number of hours. Knowing the number of occupants in the ventilated airspace $(n)$ and the fraction of indoor air that is exhaled-breath $(f)$ captured by Equation (2), we can use Equation (6) to estimate $R(I)$.

The key parameters in the simplest epidemiological model are the transmission parameter $\beta$ and the recovery rate $v .^{(13)}$ The transmission parameter $(\beta)$ represents the probability that an infective person will have contact with, and successfully infect, a susceptible person. Specifically, the transmission parameter denotes the rate at which a susceptible population becomes infected and is the expected number of new infections per infectious person per susceptible person per day. The recovery rate $(v)$ is the reciprocal of the infectious period $(1 / v)$ and is the expected number of recoveries per infected person per day. We intend to employ a standard SIR structure in the basic epidemiological model to relate $\mathrm{CO}_{2}$-based risk equation-derived $R_{0}$ to a transmission parameter $\beta$ to implicate the relationships between indoor carbon dioxide concentration and contact rate by the equation, ${ }^{(13)}$

$$
\beta=\frac{R_{0}(\nu+\mu)}{n},
$$

where $\mu$ is the birth rate and death rate of infected population $\left(\mathrm{d}^{-1}\right)$.

\section{RESULTS}

\subsection{Infected Probability and Quantum Generation Rate of Influenza}

Fig. 1 shows a histogram of epidemic curve for cases reported in terms of infected rate, the optimized distributions of infected probability, and estimated quantum generation rate of influenza. The infected probability of influenza was adapted from school-based surveillance weekly reports during the period from 2003 to 2004 by Center for Disease Control, Taiwan. Log-normal distribution that optimizes the K-S statistics shows $\mathrm{LN}(0.006,1.53)$ for infected probability (Fig. 1B) in that the selected distribution type and parameters were based on statistical criteria, comparisons of distribution parameters, and visual interpretation of histograms. Quantum generation rate of influenza can be estimated from Equation (3) with known infected probability (Fig. 1B) and adopted input parameters (Table I). After optimizing the K-S statistics, $\mathrm{LN}\left(66.91\right.$ quanta $\left.\mathrm{h}^{-1}, 1.53\right)$ was fitted for quantum generation rate of influenza (Fig. 1C).

\subsection{Risk of Outbreak of Influenza}

As an example of how Equation (6) can be employed for risk assessment, we consider a highly infectious environmental setting of aircraft, e.g., Boeing 737 (Table II). We used our proposed transmission model to quantify the uncertainty concerning $R_{0}$ attributable to the large variance of infected probability and quantum generation rate of influenza (Figs. 1B and 1C). The Monte Carlo simulation result shows the optimized log-normal distribution with geometric mean 10.35 and geometric standard deviation $1.48(\mathrm{LN}(10.35,1.48))$ is the most suitable fitted 


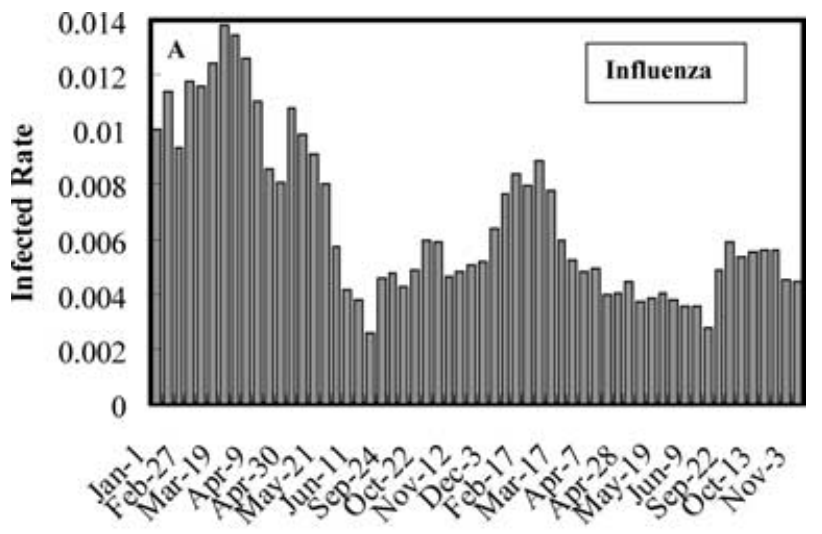

Date of Onset
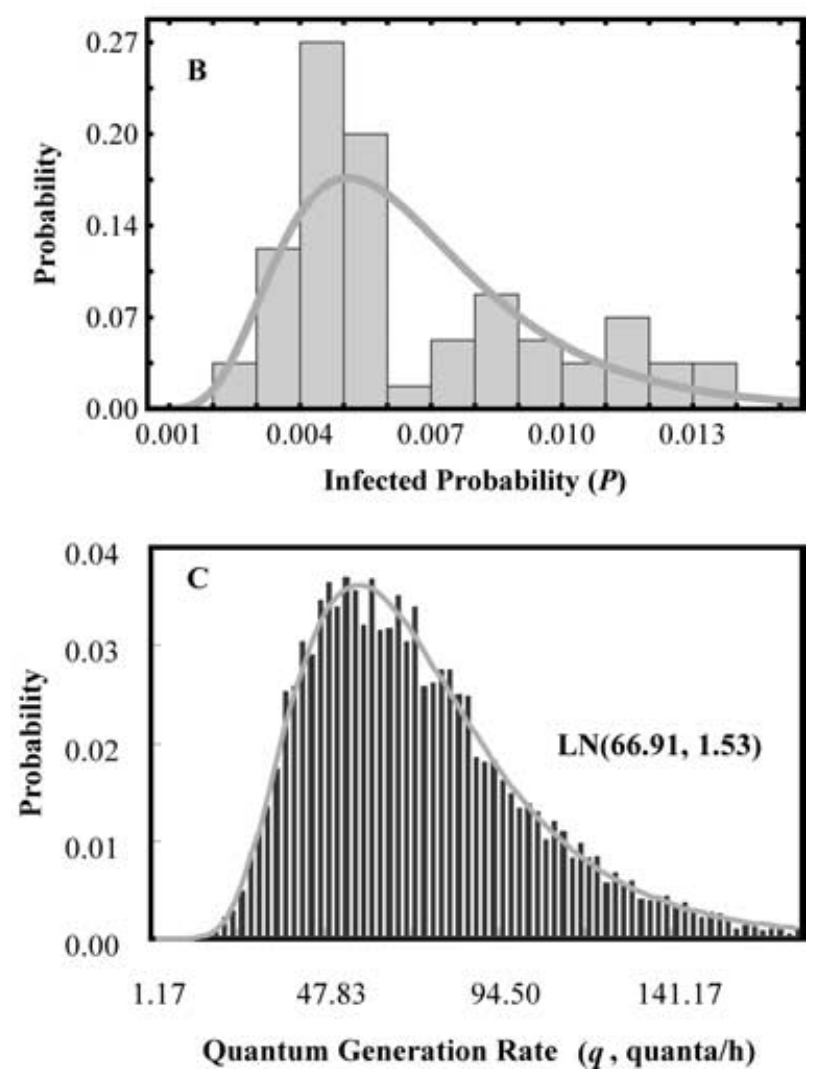

Fig. 1. Quantitative epidemiology of influenza as reported from elementary school in Taiwan during 2003-2004: (A) epidemic curve for cases reported in terms of infected rate, (B) infected probability based on Fig. 1A with fitted log-normal distribution, and (C) fitted log-normal distribution of quantum generation rate.

distribution for $R_{0}$ (Fig. 2A). We found that the distribution of $R_{0}$ is considerable right skew with wide credible intervals. Fig. 2B shows the estimate for the total proportion of infected population $(I)$ that depended only on $R_{0}$ via the expression in Equation (5), indicating the fact that the expectations based on $R_{0}$ assume a homogeneous population in direct contact.

The plotted probability calculated from the outcome of the Monte Carlo simulation followed a joint probability function (Equation (6)) describing the risk of an outbreak of influenza in a susceptible population for a range of $R_{0}$ (Fig. 2C). The cumulative density function (CDF) of $\Phi\left(R_{0}\right)$ can then be illustrated in Fig. 2D. We can further calculate the exceedence risk of $R_{0}$ through complementary CDF of $\Phi\left(R_{0}\right)$, as shown in Fig. 2E. Fig. 2E indicates that the exceedence probability of an outbreak in a Boeing 737 aircraft from a single introduction decreases with $R_{0}$, reaching about a risk that is equal to 0.5 when $R_{0}=10$. This indicates that there is a $50 \%$ probability of $R_{0}$ being bigger than 10 during an outbreak of influenza in a Boeing 737 aircraft.

\subsection{Infected Probability and Quantum Generation Rate of SARS}

The infected probability of SARS was derived from number of cases divided by total susceptible by date of symptom onset for SARS outbreak in Taipei Municipal Ho-Ping Hospital during the period from April 24, 2003 to May 8, 2003 (Figs. 3A and 3B). Infected probability (Fig. 3B) was calculated from cases divided by total population at each date of onset. The quantum generation rate (Fig. 3C) was estimated by employing the modified Wells-Riley equation associated with the fitted infected probability for SARS epidemics in Taipei Municipal Ho-Ping Hospital (Fig. 3B) and adopted input parameters (Table I). Log-normal distribution optimizing the K-S statistics showed $\operatorname{LN}(0.00015,2.64)$ for infected probability (Fig. 3B) and $\mathrm{LN}\left(28.77\right.$ quanta $\left.\mathrm{h}^{-1}, 2.64\right)$ for quantum generation rate of SARS (Fig. 3C).

\subsection{Risk of Outbreak of SARS}

We consider two highly infectious environmental settings, National Taiwan University (NTU) Hospital and an elementary school (Table II), to appraise the risk of being exposed to SARS virus. We used Taipei Municipal Ho-Ping Hospital based epidemic curve and our proposed stochastic transmission model to quantify the uncertainty concerning $R_{0}$ attributable to the large variance of infected probability and quantum generation rate of SARS (Figs. 3B and 3C). Fig. 4A illustrates probability density functions of the optimized log-normal distributions of $R_{0}$ in that the Monte Carlo simulation results show $\mathrm{LN}(2.61$, 2.61) and $\operatorname{LN}(0.77,2.61)$ are the most suitable fitted 
Table I. Input Parameters Used in Modified Wells-Riley Equation to Estimate Infected Probability from Reported Epidemiological Data and Epidemic Curves for Influenza Epidemics in Elementary School and SARS in Taipei Municipal Ho-Ping Hospital

\begin{tabular}{|c|c|c|c|c|c|c|}
\hline Settings & $\begin{array}{l}\text { People in the } \\
\text { Ventilated } \\
\text { Airspace } \\
(n)\end{array}$ & $\begin{array}{l}\text { Volume of } \\
\text { the Shared } \\
\text { Airspace } \\
\left(V \text { in } \mathrm{m}^{3}\right)\end{array}$ & $\begin{array}{c}\text { Total } \\
\text { Exposure } \\
\text { Time } \\
(t \text { in hours })\end{array}$ & $\begin{array}{c}\text { Breathing } \\
\text { Rate }^{\mathrm{a}} \\
\left(p \text { in } \mathrm{m}^{3} \mathrm{~h}^{-1}\right)\end{array}$ & $\begin{array}{l}\text { Fraction of Indoor } \\
\text { Air that is } \\
\text { Exhaled Breath } \\
(f)\end{array}$ & $\begin{array}{l}\text { Number of } \\
\text { Infectors } \\
(i)\end{array}$ \\
\hline \multicolumn{7}{|l|}{ Influenza } \\
\hline Elementary school & 50 & 600 & 6 & 0.38 & $0.00475^{\mathrm{b}}$ & 1 \\
\hline \multicolumn{7}{|l|}{ SARS } \\
\hline $\begin{array}{l}\text { Taipei Municipal } \\
\text { Ho-Ping Hospital }\end{array}$ & 20,832 & 145,602 & 6 & 1.375 & $0.0247^{\mathrm{c}}$ & 1 \\
\hline
\end{tabular}

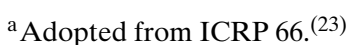

${ }^{\mathrm{b}}$ Calculated based on Equation (2) for $Q=20 \mathrm{~m}^{3} \mathrm{~h}^{-1} \mathrm{~m}^{-2}$ and floor area $=200 \mathrm{~m}^{2}$ (Construction and Planning Agency, Ministry of Interior, ROC).

${ }^{\mathrm{c}}$ Based on $Q=5$ air changes per hour $(\mathrm{ACH})$ (Construction and Planning Agency, Ministry of Interior, ROC).

distributions of $R_{0}$ for NTU Hospital and the elementary school, respectively. The most notable feature of the log-normal distribution of $R_{0}$ is the considerable right skew.

The plotted probability calculated from the outcome of the Monte Carlo simulation followed a joint probability function (Equation (5)) describing the risk of an outbreak of SARS in a susceptible population for a range of $R_{0}$ (Fig. 4C). The CDF of $\Phi\left(R_{0}\right)$ and the exceedence risk of $R_{0}$ can then be illustrated in Figs. 4D-4E. Fig. 4E indicates that the exceedence probabilities of outbreak in NTU Hospital and the elementary school from a single introduction, reached a risk about equal to 0.5 when $R_{0}=2$ and 3.8, respectively. This indicates a $50 \%$ probability that $R_{0}$ is greater than 2 during an outbreak of SARS in the elementary school, and $R_{0}$ is greater than 3.8 in NTU Hospital. Thus, it is apparent that the environmental setting plays a major role in the spread of infection.

\subsection{Relationship Between Indoor $\mathrm{CO}_{2}$ Level and Contact Rate}

A key advantage of transmission modeling is that alternative control scenarios can be examined, giving insight into the potential strategies. Here, we incorporated the $\mathrm{CO}_{2}$-based risk equation derived $R_{0}$ into a standard SIR model to assess the risk of exhaledbreath exposure in a ventilated airspace in terms of the relationship between contact rate and indoor $\mathrm{CO}_{2}$

Table II. Input Parameters Used to Estimate Basic Reproductive Number $\left(R_{0}\right)$ for Influenza in a Commercial Airliner and SARS Transmission in Hospital and Elementary School

\begin{tabular}{|c|c|c|c|c|c|c|c|}
\hline Settings & $\begin{array}{l}\text { People in } \\
\text { the Ventilated } \\
\text { Airspace } \\
(n)\end{array}$ & $\begin{array}{l}\text { Volume of } \\
\text { the Shared } \\
\text { Air Space } \\
\left(V \text { in } \mathrm{m}^{3}\right)\end{array}$ & $\begin{array}{l}\text { Total } \\
\text { Exposure } \\
\text { Time } \\
(t \text { in hours })\end{array}$ & $\begin{array}{c}\text { Breathing } \\
\text { Rate }^{\mathrm{a}} \\
\left(p \text { in } \mathrm{m}^{3} \mathrm{~h}^{-1}\right)\end{array}$ & $\begin{array}{c}\text { Estimated } \\
\text { Quantum } \\
\text { Generation Rate } \\
\left(q \text { in } \mathrm{h}^{-1}\right)\end{array}$ & $\begin{array}{l}\text { Fraction of Indoor } \\
\text { Air that is } \\
\text { Exhaled Breath } \\
(f)\end{array}$ & $\begin{array}{l}\text { Number of } \\
\text { Infectors } \\
(i)\end{array}$ \\
\hline $\begin{array}{l}\text { Influenza } \\
\text { Boeing } 737 \text { aircraft }\end{array}$ & $54^{\mathrm{b}}$ & $168^{\mathrm{b}}$ & 6 & 0.48 & $\mathrm{LN}(66.91,1.53)^{\mathrm{c}}$ & $0.0306^{\mathrm{d}}$ & 1 \\
\hline $\begin{array}{l}\text { SARS } \\
\text { National Taiwan } \\
\text { University Hospital }\end{array}$ & 300 & 7465.5 & 24 & 0.48 & $\mathrm{LN}(28.77,2.54)$ & $0.00386^{\mathrm{e}}$ & 1 \\
\hline Elementary school & 50 & 600 & 6 & 0.38 & $\operatorname{LN}(28.77,2.54)$ & $0.00475^{\mathrm{f}}$ & 1 \\
\hline
\end{tabular}

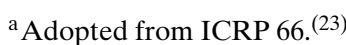

${ }^{\mathrm{b}}$ Adopted from Rudnick and Milton (2003). ${ }^{(9)}$

${ }^{c}$ Log-normal distribution with a geometric mean and a geometric standard deviation.

${ }^{\mathrm{d}}$ Based on $Q=0.3 \mathrm{ACH}$, a mean value adopted from Rudnick and Milton (2003). ${ }^{(9)}$

${ }^{\mathrm{e}} \mathrm{B}$ ased on $Q=5 \mathrm{ACH}$ (Construction and Planning Agency, Ministry of Interior, ROC).

${ }^{\mathrm{f}}$ See Table I. 

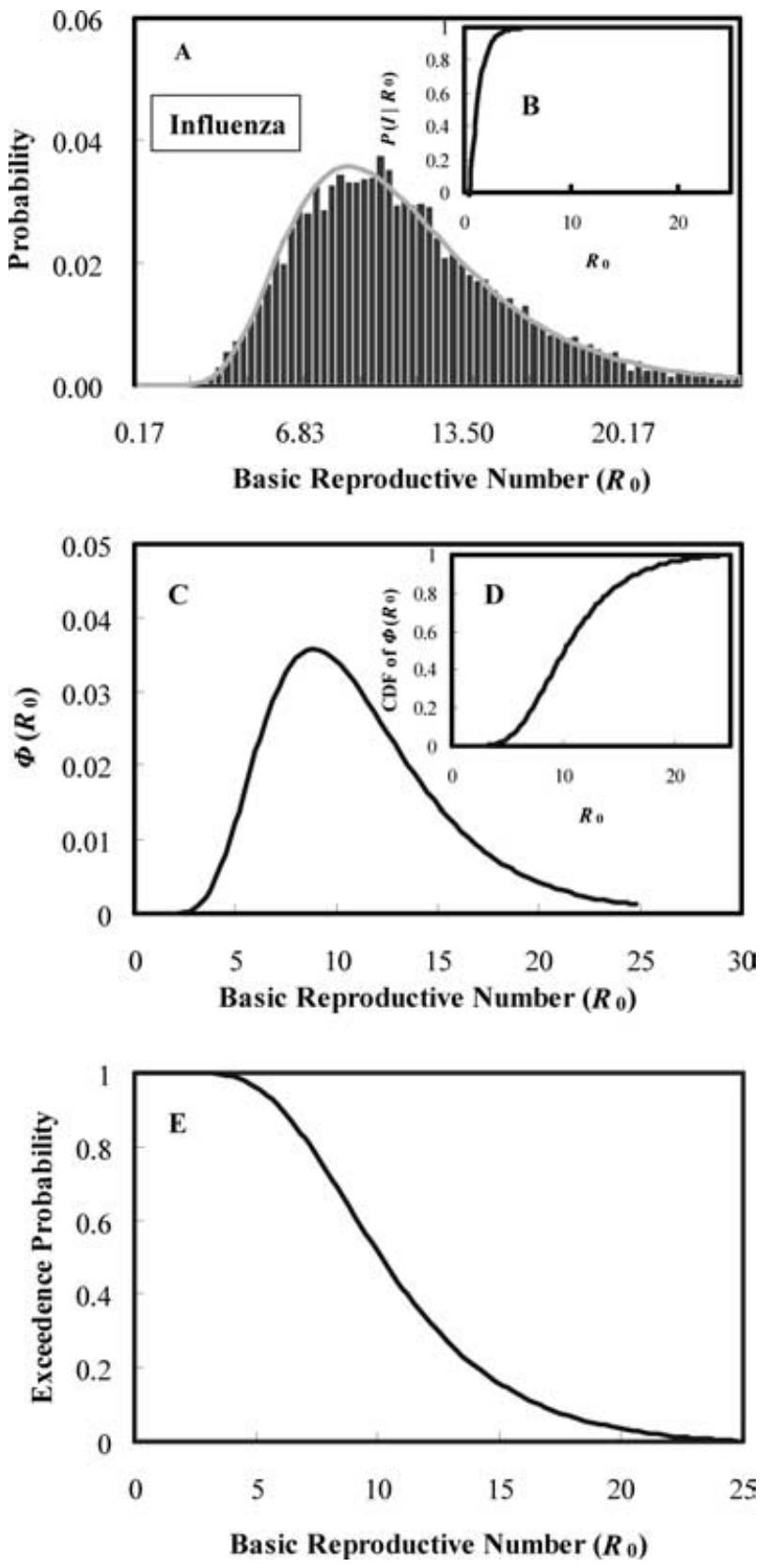

Fig. 2. Airborne infection with influenza in a Boeing 737 aircraft: (A) fitted log-normal distribution of basic reproductive number $\left(R_{0}\right),(\mathrm{B})$ total proportion of infected population $(I)$ during an epidemic, $(C)$ the risk of an outbreak of influenza in a susceptible population for a range of values of $R_{0},(\mathrm{D})$ cumulative density function of $\Phi\left(R_{0}\right)$, and (E) the exceedence probability of an outbreak of influenza in a susceptible population for a range of values of $R_{0}$.

level. Figs. 5A and 5B show the optimal fitted log-normal distributions of contact rates for influenza and SARS calculated from 10,000 iterations of Monte Carlo simulation based on Equation (7),
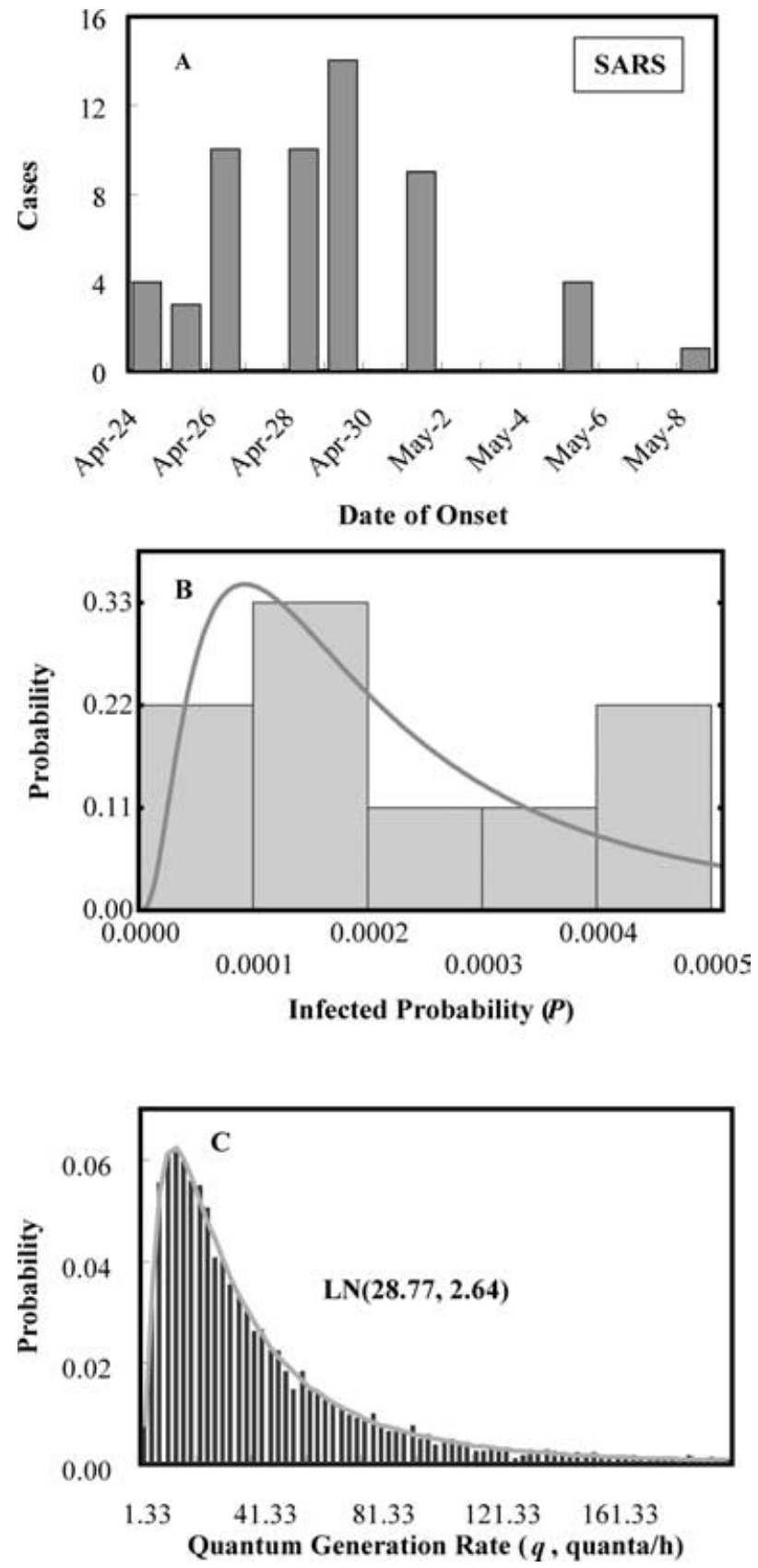

Fig. 3. Quantitative epidemiology of SARS as reported from Taipei Municipal Ho-Ping Hospital in Taiwan during April 24-May 8, 2003: (A) epidemic curve for cases reported up to May 8, 2003, (B) infected probability based on Fig. 2A with fitted log-normal distribution, and (C) fitted log-normal distribution of quantum generation rate.

where the distributions of $R_{0}$ are shown in Figs. 2A and $4 \mathrm{~A}$ and the input data of birth and recovery rates used in the SIR model are listed in Table III. The simulation outcomes that give the optimal log-normal 

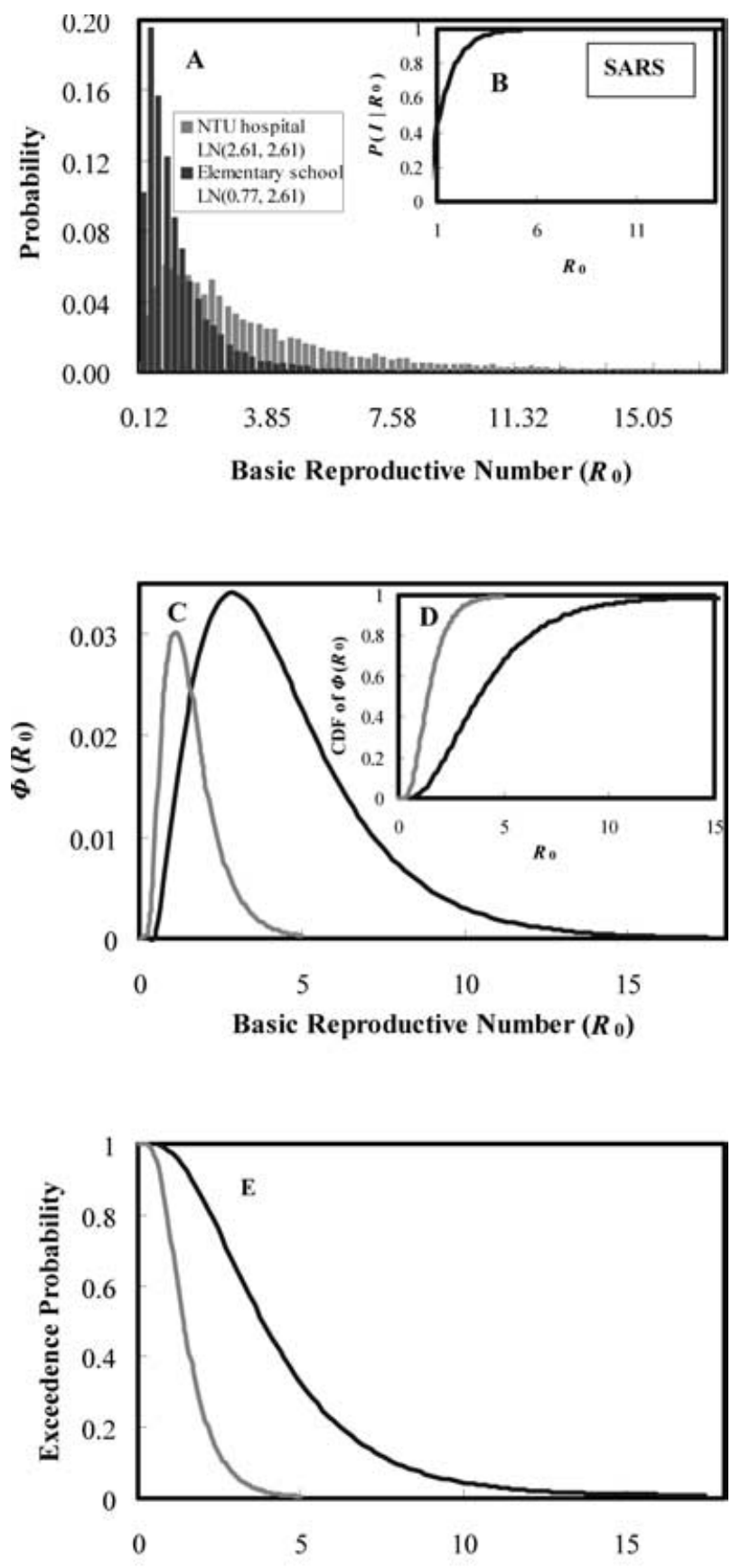

Basic Reprodutive Number $\left(\boldsymbol{R}_{0}\right)$

Fig. 4. Airborne infection with SARS in NTU Hospital and elementary school: (A) fitted log-normal distribution of basic reproductive number $\left(R_{0}\right),(\mathrm{B})$ total proportion of infected population (I) during an epidemic, (C) the risk of an outbreak of SARS in a susceptible population for a range of values of $R_{0}$. (D) cumulative density function of $\Phi\left(R_{0}\right)$, and (E) the exceedence probability of an outbreak of SARS in a susceptible population for a range of values of $R_{0}$.
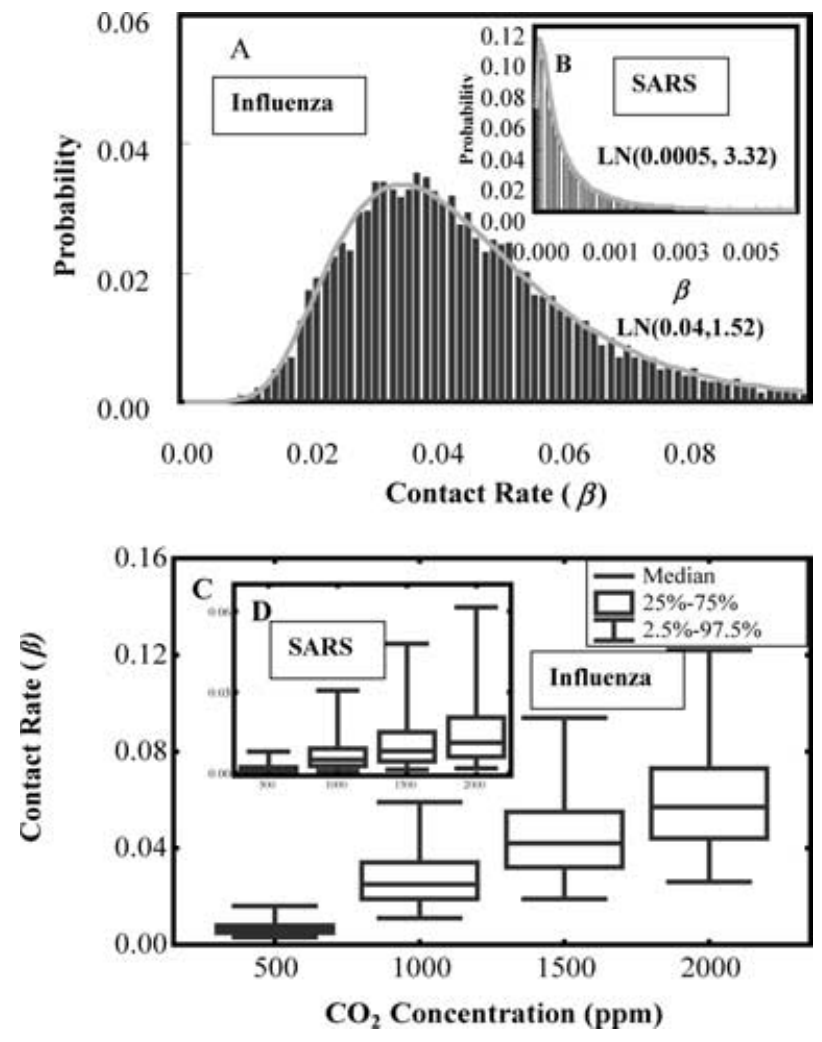

Fig. 5. Optimal fitted log-normal distributions of contact rate $(\beta)$ for (A) influenza transmission in a Boeing 737 aircraft and (B) SARS transmission in NTU Hospital. The relationships between indoor $\mathrm{CO}_{2}$ concentration and contact rate $(\beta)$ for influenza in a Boeing 737 aircraft (C) and SARS in NTU Hospital (D) are also shown in that the box and whisker plots represent the uncertainty calculated from 10,000 iterations of Monte Carlo simulation.

distributions of contact rate are $\operatorname{LN}(0.04,1.52)$ for influenza and $\mathrm{LN}(0.0005,3.32)$ for SARS (Figs. 5A and $5 \mathrm{~B}$ ).

Figs. 5C and 5D show the relationships between indoor $\mathrm{CO}_{2}$ concentration and contact rate for influenza and SARS, respectively, simulated with parameters estimated for a Boeing 737 aircraft and NTU Hospital epidemics (Table II). Figs. 5C and $5 \mathrm{D}$ also illustrate that reductions in indoor $\mathrm{CO}_{2}$ levels by increased outdoor air ventilation can achieve some reduction in transmission, although on their own they are insufficient to control influenza or SARS; other measures are also needed, such as improved infection control in a hospital, voluntary reductions in population contact rate, and movement restriction. Alternatively, if we estimate the distributions of contact rate from measured indoor $\mathrm{CO}_{2}$ concentration based on Figs. 5C and 
Table III. Birth Rate $\left(\mu, \mathrm{d}^{-1}\right)$ and Recovery Rate $\left(v, \mathrm{~d}^{-1}\right)$ Used in the SIR Model for Influenza and SARS

\begin{tabular}{lcc}
\hline Parameter & Estimate & $95 \%$ CI \\
\hline SARS & & \\
$\mu$ & $3.43 \times 10^{-2 \mathrm{a}}$ & $3.20 \times 10^{-3}-6.54 \times 10^{-2}$ \\
$v$ & $9.60 \times 10^{-2 \mathrm{a}}$ & $4.10 \times 10^{-2}-1.51 \times 10^{-1}$ \\
$\quad$ & \\
$\quad$ Influenza & & Not available \\
$\mu$ & $3.91 \times 10^{-5 \mathrm{~b}}$ & $1.92 \times 10^{-1}-4.35 \times 10^{-1}$ \\
$v$ & $2.78 \times 10^{-1 \mathrm{c}}$ & \\
\hline
\end{tabular}

${ }^{a}$ Adopted from Wang (2003). ${ }^{(24)}$

${ }^{\mathrm{b}}$ Adopted from Anderson and May (1991). ${ }^{(13)}$

${ }^{c}$ Adapted from Cauchemez et al. (2004). ${ }^{(25)}$

$5 \mathrm{D}, R_{0}$ can be directly calculated from SIR model without employing the modified Wells-Riley equation. Furthermore, the indoor $\mathrm{CO}_{2}$ concentrations might be recorded as a surrogate for outdoor air supply in that we could enhance building ventilation to reduce the population contact rate to achieve $R_{0} \leq 1$ based on different exposure times (Fig. 6).

\section{DISCUSSION}

In an environmental setting there are many factors that affect the spread of indoor airborne infection that are not included in the model. ${ }^{(3)}$ Hence, the estimate of $R_{0}$ obtained by modeling, no matter how inaccurate, can only be used as a guideline. The model, however, is more informative than a single statistic by considering the distribution of $R_{0}$ values to show uncertainty or heterogeneity, which provides insight into the epidemic process. Furthermore, a model has been developed that allows the consequences of the spread of viral or bacterial infection through a susceptible population to be quantified. The model demonstrates the risk of an outbreak in a susceptible population for a range of values of $R_{0}$. The total number of people infected can be estimated. The model can be used to investigate the effects of building ventilation for achieving the control of the spread of infectious disease. It provides a powerful tool for investigating the costs, benefits, and risks associated with control strategies for specific diseases.

Our analysis emphasizes that the risks for indoor airborne infection are determined not only by the virulence of the agent but also by environmental factors, e.g., room size and ventilation rate, and host factors, e.g., breathing rate and exposure time. Little can be done to change one's breathing rate, yet our data suggest that risks for airborne respiratory infec-
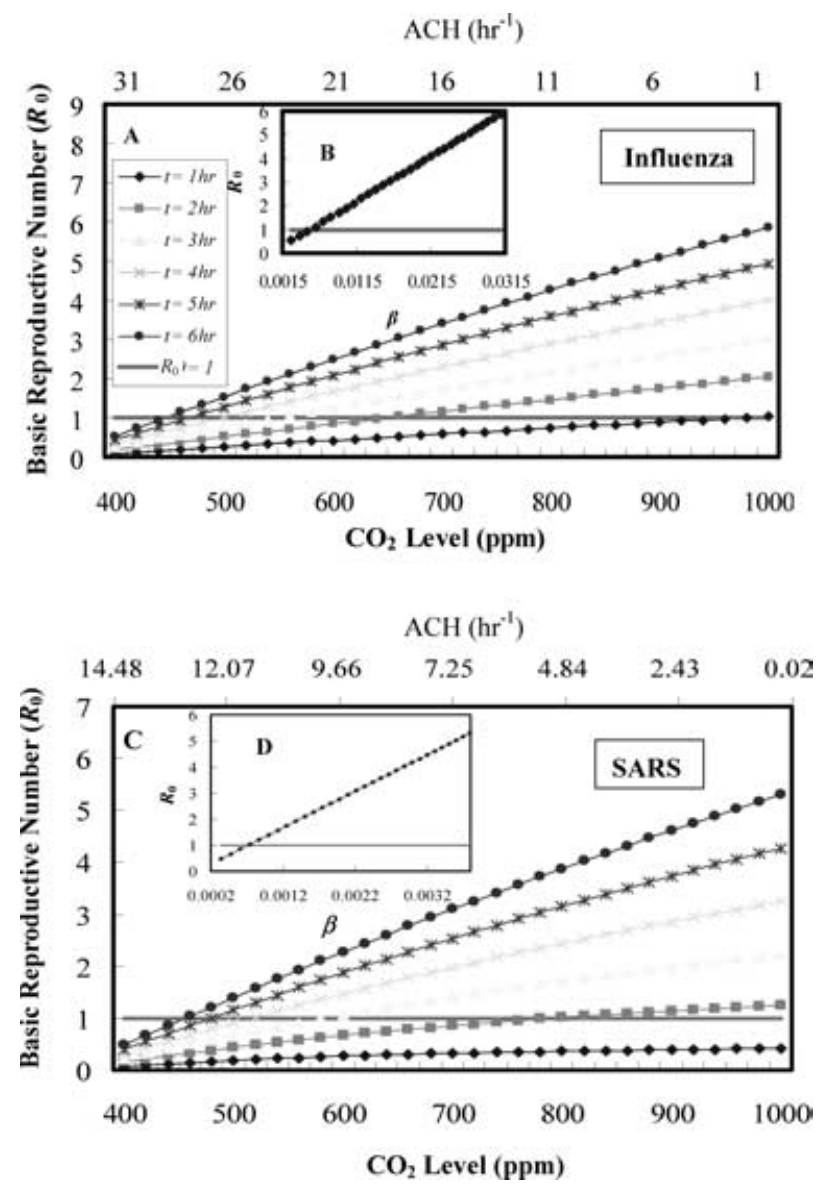

Fig. 6. Enhanced air changes per hour $(\mathrm{ACH})$ in reducing indoor $\mathrm{CO}_{2}$ concentration and contact rate may be effective in limiting the spread of airborne infection based on different exposure times $t=$ 1-6 hours: (A, B) influenza in elementary school and (C, D) SARS transmission in the hospital.

tious disease are likely to decrease considerably with fairly modest increase in room ventilation with lowconcentration exposures. Our data might be helpful in refining both public and risk manager strategies to reduce risks from indoor infection. Increased outdoor air supply rate may reduce the risk of transmitting airborne pathogens, Nardell et al., ${ }^{(20)}$ however, indicated that building ventilation may be inherently limited in decreasing this risk, especially when the concentration of exposure to infection is high. Modeling studies of transmission of indoor airborne infection have usually assumed a single uniform quantum generation rate in infected persons as well as a homogeneous concentration of infectious aerosol over time and space,,$^{(1,9,20,21)}$ but we have added a border range of values to assess the relative importance of variability in infectious aerosol production rates. 
The key advantage of employing the Wells-Riley model is that data generated by the Wells-Riley model agree with observed data in airborne infection with measles and tuberculosis. ${ }^{(8,22)}$ No other models of airborne infection with more specified variables and more complex mathematical forms have been validated against epidemiological data of any airborne infection..$^{(2,12,15)}$ Our possible approach to extending the Wells-Riley model in the future would incorporate a stochastic epidemiological model, e.g., the probabilistic SIR model. With the current model formulation, we can detect the indoor airborne infection and its relation to outdoor air supply in the indoor building environments. The indoor $\mathrm{CO}_{2}$ concentrations might be recorded as a surrogate for outdoor air supply. We could adjust the outdoor air supply rates to reduce the population contact rate that can be estimated from the model regarding the relationship between contact rate and indoor $\mathrm{CO}_{2}$ level.

Our simulation results suggest that occupants in buildings with low outdoor air supply may have an increased risk of exposure to airborne infectious droplet nuclei emanating from an infected occupant. The Wells-Riley model also allows for environmental considerations, and it can be adapted to variable exposure scenarios by stratifying by persons sharing a common dose. Epidemiological data will likely never become available to test the validity of any of these models, yet our analysis demonstrates how the risk for indoor airborne infection is determined not only by the virulence of the organism but also by the balance between infectious quantum generation rate, breathing rate, duration of exposure, and host susceptibility factors. Ventilation of the indoor environment is an important determinant of the risk for infection. Enhanced room ventilation and other engineering control measures associated with respiratory protection or public health interventions may be used to decrease the risk for infection. ${ }^{(1,2,17,18)}$ Our modeling data emphasize the need to better understand the complex interactions among host susceptibility factors, environmental factors, transmission mechanisms, and dose-response relationships in determining the risk of airborne infection.

In summary, we have used mathematical models of indoor airborne infection that are generally applicable to estimate the infection of influenza and SARS from the rate of increase of cases, to assess the likelihood of an outbreak when a case is introduced into a susceptible population, and to quantify risk profiles associated with various environmental settings.

\section{REFERENCES}

1. Barnhart, S., Sheppard, L., Beaudet, N., Stover, B., \& Balmes, J. (1997). Tuberculosis in health care settings and the estimated benefits of engineering controls and respiratory protection. Journal of Occupational and Environmental Medicine, 39, 849-885.

2. Nazaroff, W. W., Nicas, M., \& Miller, S. L. (1998). Framework for evaluating measures to control nosocomial tuberculosis transmission. Indoor Air, 8, 205-218.

3. Beggs, C. B. (2003). The airborne transmission of infection in hospital buildings: Fact or fiction? Indoor and Built Environment, 12, 9-18.

4. Ko, G., Thompson, K. M., \& Nardell, E. A. (2004). Estimation of tuberculosis risk on a commercial airliner. Risk Analysis, 24, 379-388.

5. Myatt, T. A., Johnston, S. L., Zuo, Z. F., Wand, M., Kebadze, T., Rudnick, S., \& Milton, D. K. (2004). Detection of airborne rhinovirus and its relation to outdoor air supply in office environments. American Journal of Respiratory and Critical Care Medicine, 169, 1187-1190.

6. Morens, D. M., Folkers, G. K., \& Fauci, A. S. (2004). The challenge of emerging and re-emerging infectious diseases. Nature, 430, 242-249.

7. Wells, W. F. (1934). On airborne infection. Study: II. Droplet nuclei. American Journal of Hygiene, 20, 611-618.

8. Riley, E. C., Murphy, G., \& Riley, R. L. (1978). Airborne spread of measles in a suburban elementary school. American Journal of Epidemiology, 107, 421-432.

9. Rudnick, S. N., \& Milton, D. K. (2003). Risk of indoor airborne infection transmission estimated from carbon dioxide concentration. Indoor Air, 13, 237-245.

10. Dushoff, J., \& Levin, S. (1995). The effects of population heterogeneity on disease invasion. Mathematical Biosciences, 128, 25-40.

11. Dushoff, J. (1999). Host heterogeneity and disease endemicity: A moment-based approach. Risk of indoor airborne infection transmission estimated from carbon dioxide concentration. Theoretical Population Biology, 56, 325-335.

12. Nicas, M., \& Hubbard, A. (2002). A risk analysis for airborne pathogens with low infectious doses: Application to respirator selection against coccidioides immitis spores. Risk Analysis, 22, 1153-1163.

13. Anderson, R. M., \& May, R. M. (1991). Infectious Disease of Humans: Dynamics and Control. Oxford: Oxford University Press.

14. Diekmann, O., \& Heesterbeck, J. A. P. (2002). Mathematical Epidemiology of Infectious Diseases: Model Building, Analysis and Interpretation. New York: Wiley.

15. Ferguson, N. M., Keeling, M. J., Edmunds, W. J., Gani, R., Grenfell, B. T., Anderson, R. M., \& Leach, S. (2003). Planning for smallpox outbreak. Nature, 425, 681-685.

16. Eisenberg, J. N. S., Brookhart, M. A., Rice, G., Brown, M., \& Colford, J. M. (2002). Disease transmission models for public health decision making: Analysis of epidemic and endemic conditions caused by waterborne pathogens. Environmental Health Perspectives, 110, 783-790.

17. Lipsitch, M., Cohen, T., Cooper, B., Robins, J. M., Ma, S., James, L., Gopalakrishna, G., Chew, S. K., Tan, C. C., Samore, M. H., Fisman, D., \& Murray, M. (2003). Transmission dynamics and control of severe acute respiratory syndrome. Science, 300, 1966-1970.

18. Riley, S., Fraser, C., Donnelly, C. A., Ghani, A. C., AbuRaddad, L. J., Hedley, A. J., Leung, G. M., Ho, L. M., Lam, T. H., Thach, T. Q., Chau, P., Chan, K. P., Leung, P. Y., Tsang, T., Ho, W., Lee, K. H., Lau, E. M. C., Ferguson, N. M., \& Anderson, R. M. (2003). Transmission dynamics of the etiological agent of SARS in Hong Kong: Impact of public health interventions. Science, 300, 1961-1966. 
19. Gani, R., \& Leach, S. (2001). Transmission potential of smallpox in contemporary populations. Nature, 414, 748-751.

20. Nardell, E. A., Keegan, J., Cheney, S. A., \& Etkind, S. C. (1991). Airborne infection. Theoretical limits of protection achievable by building ventilation. American Review of Respiratory Disease, 144, 302-306.

21. Fennelly, K. P., Davidow, A. L., Miller, S. L., Connel, N., \& Ellner, J. L. (2004). Airborne infection with Bacillus anthracis-From mills to mail. Emerging Infectious Diseases, 10, 996-1001.

22. Wells, W. F. (1955). Airborne Contagion and Air Hygiene: An Ecological Study of Droplet Infection. Cambridge, MA: Harvard University Press.
23. ICRP. (1994). Human Respiratory Tract Model for Radiological Protection, a Report of Task Group of the International Commission on Radiological Protection, ICRP Publication, Vol. 66. New York: Elsevier.

24. Wang, Y. C. (2003). Mathematical Model for Estimating Reproductive Number $\left(R_{0}\right)$ of Infectious Disease: Two Illustrations Using Endemic Hepatitis B Infection and Epidemic Spread of Severe Acute Respiratory Syndrome (SARS). National Taiwan University.

25. Cauchemez, S., Carrat, F., Viboud, C., Valleron, A. J., Boelle, P. Y., \& Bayesian, A. (2004). MCMC approach to study transmission of influenza: Application to household longitudinal data. Statistics in Medicine, 23, 3469-3487. 